\title{
In the race at last: post-hoc analysis of GALATHEA and TERRANOVA
}

A revolution is occurring in the world of medicinethe precision medicine revolution. ${ }^{1}$ The goal is to give patients with the right characteristics therapy that is precisely targeted to their disease pathology with the expectation of efficacy and minimal harmful effects. In airway diseases, the clinicians' goal should be to never have to prescribe oral corticosteroids again. In this, respiratory medicine is lagging behind many other specialities, but this field is now through the start line. The efficacy of monoclonal antibodies against the interleukin-5 (IL-5) pathway in asthma has been shown ${ }^{2}$ raising the hopes of achieving similar results in chronic obstructive pulmonary disease (COPD). The phenotype to target is a raised blood eosinophil count. This phenotype exists in COPD as well as asthma (up to $40 \%$ of patients) and predicts risk of exacerbation.

Thus far, the results of clinical trials have been mixed. Mepolizumab (anti-IL-5 antibody), has shown a small improvement in annual COPD exacerbation rate when versus placebo in a phase 3 trial (METREX), but these data were not replicable in the parallel METREO study. ${ }^{3}$ Benralizumab (antibody against the alpha chain of the IL-5 receptor) in the initial GALATHEA and TERRANOVA studies did not reach its primary endpoints in reducing exacerbations versus placebo. ${ }^{4}$

Gerard Criner and colleagues ${ }^{5}$ have pooled the 3910 patients in both the GALATHEA and TERRANOVA studies to investigate if any patient characteristics did predict response to benralizumab. ${ }^{5}$ This analysis is to be viewed with caution and should be considered as hypothesis-generating rather than definitive. However, the data generated might be invaluable for the future.

A response to benralizumab only occurred at the $100 \mathrm{mg}$ dose. The three clinical characteristics that suggested the greatest response to benralizumab were lower baseline lung function, bronchodilator response, and higher baseline exacerbation history. Higher concentrations of blood eosinophils were predictive of response if associated with at least three or more previous exacerbations or if the patient was using inhaled triple therapy.

These patients did not have asthma. They had traits that were predictive of response to anti-IL5 therapy, which are seen in patients with COPD, and so we recommend that these tests should be added to the routine assessment of patients with COPD and added to current guidelines.

To benefit from these new therapies, a patient needs to have not only the trait that will respond to the treatment but also the necessary risk that will be reduced. Hence the need to have a high baseline exacerbation rate as well as a high eosinophil count. This need has been shown in both treatment and treatment withdrawal studies using inhaled corticosteroids in patients with COPD. To effectively use such data, we recommend analysing events considering blood eosinophils as a continuous variable. ${ }^{6}$

The analyses of Criner and colleagues also raise some interesting questions, particularly with regard to mechanisms. Why was a higher dose required to prevent COPD exacerbations? This finding was not seen with mepolizumab. Phase 1 data for benralizumab show that the suppression of blood eosinophils is complete even with lower doses of benralizumab. Older data ${ }^{8}$ show that bronchial biopsies taken during acute exacerbations have up to a 30 times increase in eosinophils compared with patients in a stable state. Is a higher dose required to prevent the chemotaxis of eosinophils into the bronchial epithelium, and more so at the time of exacerbation?

Criner and colleagues ${ }^{5}$ accept that their study did not attempt to phenotype COPD exacerbations. METREO and METREX ${ }^{3}$ achieved numerically fewer systemic glucocorticoid treated exacerbations with anti-IL5 therapy compared with placebo. These findings suggest a possible need to try to characterise types of exacerbations. Other colleagues have shown that systemic corticosteroids can be safely withheld in COPD exacerbations without a raised blood eosinophil count. ${ }^{9,10}$ Future exacerbation studies using an antiIL5 approach should classify the subtypes of COPD exacerbations, which might vary from the baseline characteristics. No therapy has reduced exacerbation rates in a high-risk population to fewer than approximately one event a year. Exacerbations are not homogenous events and should be phenotyped in order
Lancet Respir Med 2019 Published Online September 28, 2019 https://doi.org/10.1016/PI See Online/Articles https://doi.org/10.1016/PI 
to understand their mechanisms. The results might be that only eosinophilic COPD exacerbations are being suppressed by anti-IL5 therapy with non-eosinophilic events being unaffected.

To compete in the precision medicine race, clinicians need to have clear cost-effectiveness data; these will follow. However, these data will require an absolute focus on targeting the right patient population and show reductions in hospital admissions or mortality.

In all, we congratulate Criner and colleagues on their success in hypothesis generation. We look forward to the results of the RESOLUTE study (NCT04053634), which will put these hypotheses to the test. We hope that the RESOLUTE investigators will consider subtyping the complex events that we call exacerbations in further detail to establish if a potentially significant benefit of treatment is being missed.

Sanjay Ramakrishnan, *Richard Russell

Respiratory Medicine, Nuffield Dept of Medicine, University of Oxford, Oxford OX3 7FX, UK

richard.russell@ndm.ox.ac.uk

RR is funded by the NIHR Oxford Biomedical Research Centre. We declare no competing interests

1 The White House, President Barack Obama. The precision medicine initiative. https://obamawhitehouse.archives.gov/precision-medicine (accessed Sept 18, 2019).

2 Pavord ID, Korn S, Howarth P, et al. Mepolizumab for severe eosinophilic asthma (DREAM): a multicentre, double-blind, placebo-controlled trial. Lancet 2012; 380: 651-59.

3 Pavord ID, Chanez P, Criner GJ, et al. Mepolizumab for eosinophilic chronic obstructive pulmonary disease. N EnglJ Med 2017; 377: 1613-29.

4 Criner GJ, Celli BR, Brightling CE, et al. Benralizumab for the prevention of COPD exacerbations. N Engl J Med 2019; 381: 1023-34.
5 Criner GJ, Celli BR, Singh D, et al. Predicting response to benralizumab in chronic obstructive pulmonry disease: analyses of GALATHEA and TERRANOVA studies. Lancet Respir Med 2019; published online Sept 28. https://doi:XXX [Prod: please update once known].

6 Bafadhel M, Peterson S, De Blas MA, et al. Predictors of exacerbation risk and response to budesonide in patients with chronic obstructive pulmonary disease: a post-hoc analysis of three randomised trials. Lancet Respir Med 2018; 6: 117-126.

7 Busse WW, Katial R, Gossage D, et al. Safety profile, pharmacokinetics, and biologic activity of MEDI-563, an anti-IL-5 receptor alpha antibody, in a phase I study of subjects with mild asthma. J Allergy Clin Immunol 2010; 125: $1237-44$.

8 Saetta M, Di Stefano A, Maestrelli P, et al. Airway eosinophilia in chronic bronchitis during exacerbations. Am J Respir Crit Care Med 1994; 150: 1646-52.

9 Bafadhel M, McKenna S, Terry S, et al. Blood eosinophils to direct corticosteroid treatment of exacerbations of chronic obstructive pulmonary disease: a randomized placebo-controlled trial. AmJ Respir Crit Care Med 2012; 186: 48-55.

10 Sivapalan P, Lapperre TS, Janner J, et al. Eosinophil-guided corticosteroid therapy in patients admitted to hospital with COPD exacerbation (CORTICO-COP): a multicentre, randomised, controlled, open-label, non-inferiority trial. Lancet Respir Med 2019; 7: 699-709. 